\title{
Effect of Heat Treatment on the Ultrasonic Attenuation in Mn-Ferrites
}

\author{
Y. Kawai, V.A.M. Brabers* and Z. Simsa** \\ Gakushuin University, 1-5-1 Meijiro, Tokyo 171, Japan \\ * University of Technology, 5600 Eindhoven, The Netherlands \\ ** Institute of Physics ASCR, Cukrovarnická 10, 16200 Praha 6, Czech Republic
}

\begin{abstract}
The acoustic losses in single crystals of manganese ferrites were studied in the temperature range from 180 to $650 \mathrm{~K}$ by applying longitudinal vibrations of $150 \mathrm{kHz}$. Loss peaks were observed at 232 and $537 \mathrm{~K}$ with activation energies of 0.3 and $0.8 \mathrm{eV}$, respectively. The first loss peak at $232 \mathrm{~K}$ is regarded as a stress-induced relaxation of electrons between two and three-valent ions. The second peak at $537 \mathrm{~K}$ is ascribed to the exchange motion of iron cations and their vacancies on octahedral sites. Heat treatments at 300 and $600^{\circ} \mathrm{C}$ affect the oxygen stoichiometry and distribution of cations between octahedral and tetrahedral sites which results in the changes of the loss peaks magnitudes.
\end{abstract}

\section{INTRODUCTION}

In our previous paper the classification of the magnetic and acoustic relaxation processes were suggested [1]. It appeared that many phenomena have a common origin. Namely, relaxations at low temperatures and with low activation energies were assigned to electronic processes occurring mostly between two and three valent iron ions. Relaxations at high temperatures and possessing larger activation energies are due to the exchange of cations with their neighbouring vacancies.

To get more information about the processes occurring in the intermediate temperature and energy range the acoustic loss and Young's moduli of three single crystal samples out of the manganese ferrite spinel system were investigated.

\section{EXPERIMENT}

Specimens for acoustic measurements were prepared from single crystal rods of the $\mathrm{Mn}_{\mathrm{x}} \mathrm{Fe}_{3-\mathrm{x}} \mathrm{O}_{4}(\mathrm{x}=0.82,1.0$ and 1.1$)$ spinel series grown by arc-image floating-zone melting as described elsewhere [2]. After the growth process the crystals were additionally heat-treated in controlled atmospheres to improve oxygen stoichiometry and mechanical properties.

From the single crystal rods rectangular bars of approximate dimensions of $2.5 \times 2.5 \times 20 \mathrm{~mm}^{3}$ along the main crystallographic axes $\langle 100\rangle,\langle 110\rangle$ or $\langle 111\rangle$ were cut. Corresponding (100), (110) and (211) faces were finely polished and etched by $\mathrm{HCl}$ solution to a depth of about $10 \mu \mathrm{m}$ in order to remove the outermost damaged surfaces.

Acoustic losses and Young's moduli were measured by a composite-bar resonator [3] which was mounted in a metal chamber evacuated to $10^{-2}$ torr. Measurements in the temperature range from 180 to $650 \mathrm{~K}$ were carried out in a liquid nitrogen cryostat using longitudinal vibrations mainly at $149 \mathrm{kHz}$ with a strain amplitude of $10^{-6}$. Both the mechanical stress and the external dc magnetic field up to 600 Oe were applied parallel to the longest edge of the specimen.

\section{RESULTS}

The representative temperature dependencies of the acoustic loss and the Young's modulus for the as grown samples are shown in Fig. 1 a). Two clear loss peaks accompanied with dips in the Young's moduli are found at 232 and $537 \mathrm{~K}$ for $<110>$ and $<111>$ oriented specimens. No anomalies in $<100>$ oriented samples were found in agreement with earlier results [3-4]. The high temperature loss peak possessed a nonsymmetrical shape (open symbols) which became symmetrical (closed symbols) upon the application of the external magnetic field. The nonsymmetrical contribution extends from about $380 \mathrm{~K}$ up to $545 \mathrm{~K}$, the latter temperature coinciding with the Curie temperature of the $\mathrm{Mn}_{1.1} \mathrm{Fe}_{1.9} \mathrm{O}_{4}$ sample. Similar measurements at two other resonant frequencies (130 and $201 \mathrm{kHz}$ ) showed a temperature shifts of the peak's maxima so that the pre-exponential factor $\tau_{0}$ and the activation energy $\epsilon$ in the Arrhenius relation $\tau=\tau_{0} \exp (\epsilon / \mathrm{kT})$ could be determined as $10^{-13} \mathrm{sec}$ and $0.3 \mathrm{eV}$ for the low-temperature peak and $3 \times 10^{-14} \mathrm{sec}$ and $0.8 \mathrm{eV}$ for the high-temperature one, respectively. A plot of the low temperature peak for all the as grown samples is seen in the inset of Fig. 1 a). 
Three kinds of heat treatments were carried out with the specimens in order to change the oxygen stoichiometry and to achieve a different distribution of cations among tetrahedral and octahedral positions [5]:

(1) heating at $600^{\circ} \mathrm{C}$ in air for 1 hour followed by quenching to room temperature,

(2) heating at $300^{\circ} \mathrm{C}$ in air for 1 week followed by quenching to room temperature,

(3) heating at $600^{\circ} \mathrm{C}$ in nitrogen for 1 hour and slow-cooling to room temperature.

The influence of the heat treatment procedures on sample $x=1.1$ are demonstrated in Fig. 1 b). All measurements are made in an applied magnetic field of 600 Oe and only minor changes can be detected with the air treatments (1) and (2) of the sample. On the other hand, a significant decrease of the high temperature peak and a small increase of the low temperature peak is found on reduction of the sample by treatment (3) in nitrogen atmosphere. The effect of the heat treatment upon the low temperature peak for sample $\mathrm{MnFe}_{2} \mathrm{O}_{4}$ is shown in the inset of Fig. $1 \mathrm{~b}$ ).

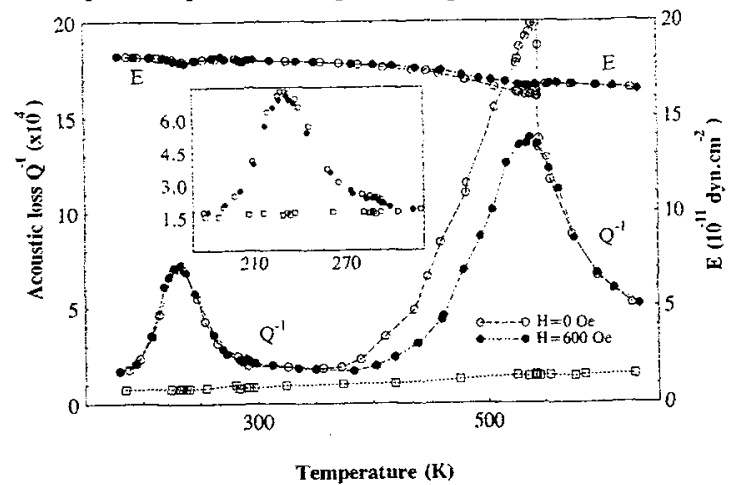

a)

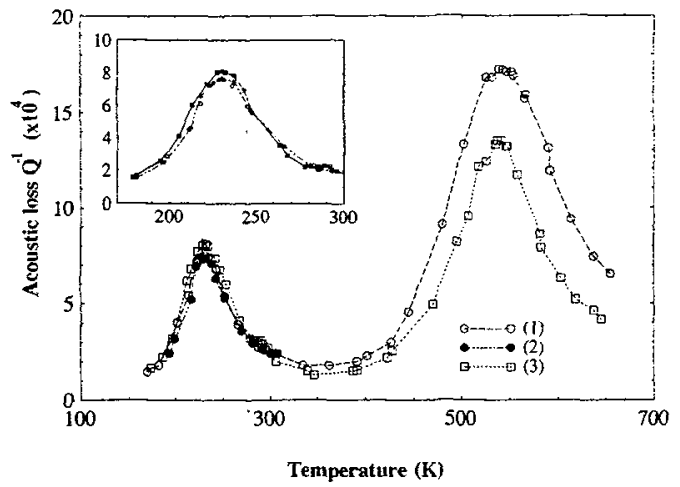

b)

Fig. 1. a) Acoustic loss $\left(\mathrm{Q}^{-1}\right)$ and Young's modulus (E) for $\mathrm{Mn}_{1,1} \mathrm{Fe}_{1,9} \mathrm{O}_{4}<111>(0 \bullet)$ and $\mathrm{MnFe}_{2} \mathrm{O}_{4}<100>(\square)$ oriented samples. In the inset the acoustic loss of all as grown samples in $\langle 111\rangle$ direction for the low temperature peak are plotted.

b) Influence of heat treatment on the acoustic loss of $\mathrm{Mn}_{1.1} \mathrm{Fe}_{1.9} \mathrm{O}_{4}$ in $\langle 111\rangle$ direction. The inset shows the influence of the heat treatments on the low temperature peak of the $\mathrm{MnFe}_{2} \mathrm{O}_{4}$ sample.

Comparing the present results with those of as-grown samples we can conclude that changes of the distribution of cations (difference between treatments (1) and (2)) hardly influence the acoustic loss of the samples. Treatments in reducing atmosphere (3) decrease magnitude of the high-temperature peak and slightly increase magnitude of the low-temperature peak.

\section{DISCUSSION}

To discuss the results we recall to our early paper [1]. The first loss peak at $232 \mathrm{~K}$ is regarded as a stress-induced relaxation of electrons between two and three-valent manganese and iron ions. The second peak at $537 \mathrm{~K}$ is ascribed to the interchange of iron cations and vacancies on octahedral sites. Heat treatments in the temperature range from 300 to $600^{\circ} \mathrm{C}$ were shown to affect the distribution of cations between octahedral and tetrahedral sites [5]. Moreover, the heat treatment in nitrogen decreases the concentration of cation vacancies on octahedral sites.

From Fig. 1 it follows that the reduction of the oxidation state (treatment (3)) has much larger influence on the acoustic losses than the changes in the degree of inversion of the samples (treatments (1) and (2)). A significant decrease of the magnitude and narrowing of the high temperature peak after reduction (3) support the idea that the relaxation processes connected with this peak are due to the cation - vacancy exchange.

A slight increase of the low temperature peak with samples $x=1$ and 1.1 and its absence for $x=0.82$ are in accord with the early idea that the processes involved are connected with the simultaneous presence of two valent iron and three valent manganese ions forming pairs in octahedral sites. For $\mathrm{x}=1$ a number of such "free" pairs attains a maximum. The number of pairs decreases with rising manganese content $\mathrm{x}$ due to a decrease of two valent octahedral ions, probably present as $\mathrm{Mn}^{2+}$. A sharp decrease of this effect below $x=1.0$ (and its total absence in $x=0.82$ sample) results from the "blocking" effect of the abundant $\mathrm{Fe}^{2+}$ for these compositions that prevent reorientation of the octahedral iron-manganese "pairs".

\section{References}

[1] Z. Šinša, R. Tesař, J. Koláček, M. Guyot, V. Cagan, M. Rouabhi and V.A.M. Brabers, "Magnetic Losses in Single Crystal Ferrites", Proceedings of ICF6, Tokyo 1992, T. Yamaguchi and M.Abe Eds. (JSPPM, Tokyo 1992) pp. 687-690.

[2] V.A.M. Brabers, J. Cryst. Growth 8 (1971) 26-29.

[3] Y. Kawai and T. Ogawa, Phys. Stat. Solidi (a) 76 (1983) 814-819.

[4] Z. Šimša, Y. Kawai and V.A.M. Brabers, Phys. Stat. Solidi (a) 88 (1985) 611-617.

[5] Z. Jirák, Z. Šimša, J. Šimšová, V. Roskovec, S. Vratislav and V.A.M. Brabers, "Magnetic Moments and the Inversion Degree of Mn-ferrite", Proceedings of ICM-73, Vol. V, Moscow 1973 (Nauka, Moscow 1974) 260-264. 